\title{
A recessive allele for delayed flowering at the soybean maturity locus E9 is a leaky allele of FT2a, a FLOWERING LOCUS T ortholog
}

Chen Zhao ${ }^{1}$, Ryoma Takeshima', Jianghui Zhu', Meilan Xu³, Masako Sato', Satoshi Watanabe², Akira Kanazawa', Baohui Liu ${ }^{3^{*}}$, Fanjiang Kong ${ }^{3^{*}}$, Tetsuya Yamada ${ }^{1}$ and Jun Abe $^{1^{*}}$

\begin{abstract}
Background: Understanding the molecular mechanisms of flowering and maturity is important for improving the adaptability and yield of seed crops in different environments. In soybean, a facultative short-day plant, genetic variation at four maturity genes, $E 1$ to $E 4$, plays an important role in adaptation to environments with different photoperiods. However, the molecular basis of natural variation in time to flowering and maturity is poorly understood. Using a cross between early-maturing soybean cultivars, we performed a genetic and molecular study of flowering genes. The progeny of this cross segregated for two maturity loci, E1 and E9. The latter locus was subjected to detailed molecular analysis to identify the responsible gene.

Results: Fine mapping, sequencing, and expression analysis revealed that E9 is FT2a, an ortholog of Arabidopsis FLOWERING LOCUS T. Regardless of daylength conditions, the e9 allele was transcribed at a very low level in comparison with the E9 allele and delayed flowering. Despite identical coding sequences, a number of single nucleotide polymorphisms and insertions/deletions were detected in the promoter, untranslated regions, and introns between the two cultivars. Furthermore, the e9 allele had a Ty1/copia-like retrotransposon, SORE-1, inserted in the first intron. Comparison of the expression levels of different alleles among near-isogenic lines and photoperiod-insensitive cultivars indicated that the SORE-1 insertion attenuated FT2a expression by its allele-specific transcriptional repression. SORE-1 was highly methylated, and did not appear to disrupt FT2a RNA processing.

Conclusions: The soybean maturity gene E9 is FT2a, and its recessive allele delays flowering because of lower transcript abundance that is caused by allele-specific transcriptional repression due to the insertion of SORE-1. The FT2a transcript abundance is thus directly associated with the variation in flowering time in soybean. The e9 allele may maintain vegetative growth in early-flowering genetic backgrounds, and also be useful as a long-juvenile allele, which causes late flowering under short-daylength conditions, in low-latitude regions.
\end{abstract}

Keywords: Maturity gene E9, FLOWERING LOCUS T, FT2a, Soybean (Glycine max), Flowering, Ty1/copia-like retrotransposon, SORE-1, Methylation

\footnotetext{
*Correspondence: liubh@neigaehrb.ac.cn; kongfj@iga.ac.cn; jabe@res.agr. hokudai.ac.jp

${ }^{3}$ The Key Laboratory of Soybean Molecular Design Breeding, Northeast Institute of Geography and Agroecology, Chinese Academy of Sciences, Harbin 150081, China

${ }^{1}$ Research Faculty of Agriculture, Hokkaido University, Sapporo, Hokkaido 060-8589, Japan

Full list of author information is available at the end of the article
} 


\section{Background}

Knowledge of molecular mechanisms of flowering and maturity is important for understanding the phenology of seed crops and for maximizing yield in a given environment. On the basis of knowledge accumulated for Arabidopsis thaliana, the molecular mechanisms of flowering have been studied in many crops. These studies have revealed common important genes, such as FLOWERING LOCUS T (FT) and CONSTANS (CO), but also their functional divergence and diversity of genetic mechanisms underlying the natural variation of flowering time within species [1-3].

Soybean (Glycine $\max$ (L.) Merrill) is a facultative short-day plant. Rich genetic variability in photoperiod responses enables the crop to adapt to a wide range of latitudes. This wide adaptability has been created by natural variations in a number of major genes and quantitative trait loci (QTLs) that control flowering [4]. Ten major genes have been identified so far to control time to flowering and maturity in soybean: $E 1$ and E2 [5], E3 [6], E4 [7], E5 [8], E6 [9], E7 [10], E8 [11], E9 [12], and J [13]. Dominant alleles at E6, E9, and $J$ promote early flowering, whereas dominant alleles at other loci delay flowering and maturity. $E 6$ and $J$ have been identified in the progeny of crosses between standard and lateflowering cultivars with a long-juvenile habit, which causes late flowering under short days [9, 13]. E9 has been identified through the molecular dissection of a QTL for early flowering introduced from a wild soybean accession [12, 14]. Molecular mechanisms that involve four of the ten genes ( $E 1$ to $E 4$ ) have been identified. E1 encodes a possible transcription factor down-regulating FT2 $a$ and FT5a (soybean FT orthologs) [15] and has the most marked effect on flowering time [16-18]. E2 is an ortholog of Arabidopsis GIGANTEA (GI) [19]. E3 and E4 encode the phytochrome A isoforms, GmPHYA3 and GmPHYA2, respectively [20, 21].

The soybean genome has at least ten FT homologs, among which six promote flowering of the Arabidopsis $f t$ mutant or ecotype Columbia (Col-0) when ectopically expressed [22-25]. Their expression profiles differ depending on tissues and growth stages, suggesting their subfunctionalization in soybean flowering [23-25]. Among the six homologs, FT2a and FT5a have been extensively studied [15, 19, 22-28], because their expression patterns closely follow photoperiodic changes [24] and their overexpression promotes flowering even under non-inductive conditions [26, 27]. The photoperiodic expression patterns of $F T 2 a$ and FT5 a are most likely controlled by $E 1$ and its homologs, E1La and E1Lb, which in turn are under the control of E3 and E4 [15, 28]. E2 inhibits FT2a expression possibly through a pathway different from the E1-PHYA pathway $[19,28]$.
Allelic variations at E1-E4 generate some but not all of the variation in flowering time among soybean cultivars $[18,29]$. Various combinations of mutations that occur independently at E1,E3, and E4 lead to insensitivity or low sensitivity of flowering to photoperiod [29, 30]. Besides the above four genes, a number of soybean orthologs of Arabidopsis flowering genes have been characterized: $C O L$ (CO-like) [25, 31], CRY (CRYPTOCHROME) [32, 33], FKF1 [34], FLD (FLOWERING LOCUS D) [35], FUL (FRUITFULL) [36], RAV-like (RELATED TO ABI3/VP1like) [37], SOC1/AGL2O (SUPPRESSOR OF OVEREXPRES SION OF COL1/AGAMOUS-LIKE 20) [38, 39], TARGET OF EAT1 (TOE) [40], and ZTL (ZEITLUPE) [41]. A genome-wide association study also revealed a number of SNPs that were significantly associated with flowering time; some of these SNPs implied an involvement of orthologs to Arabidopsis flowering genes, such as EARLY FLOWERING 8 and SOC1 or AGAMOUS-LIKE 6, in the control of flowering time in soybean [42]. However, our understanding of the roles of these orthologs in the natural variation of flowering in soybean is still limited. Jiang et al. [43] found diverse sequence variations in the FT2a promoter region among soybean cultivars, despite the coding region being highly conserved. Although some of these polymorphisms are significantly associated with variation in flowering time among the cultivars tested, their roles in FT2a expression is not fully understood [43].

In this study, using a cross between early-maturing cultivars of different origins, we found that segregation of flowering time was partly associated with a tagging marker of the maturity gene E9. We demonstrate that $E 9$ is identical to $F T 2 a$, and its recessive allele has an insertion of the Ty1/copia-like retrotransposon in the first intron, which reduces the FT2a transcript level and delays flowering.

\section{Results}

Segregation of flowering time in the progeny of a cross between Harosoy and Toyomusume

Two early-maturing cultivars, a Canadian cultivar, Harosoy (HA), and a Japanese cultivar, Toyomusume (TO), were used in the crossing. They have the same maturity genotypes at E2, E3, and E4 (e2/e2 E3/E3 E4/ $E 4)$, but differ in the $E 1$ genotype: HA has a hypomorphic e1-as allele, whereas TO has an e1-nl allele, which lacks the genomic region $(\sim 130 \mathrm{~kb})$ containing the entire E1 gene $[15,18]$. TO and HA flowered almost at the same time under natural daylength conditions in Sapporo, Japan $\left(43^{\circ} 07^{\prime} \mathrm{N}, 141^{\circ} 35^{\prime} \mathrm{E}\right)$, although the former flowered 3 to 5 days earlier than the latter. However, flowering times in the $\mathrm{F}_{2}$ population varied widely (4667 days after sowing; Fig. 1a). Since the allelic variation at $E 1$ has a large effect on flowering time, we first evaluated the effects of $E 1$ alleles on flowering time in the 


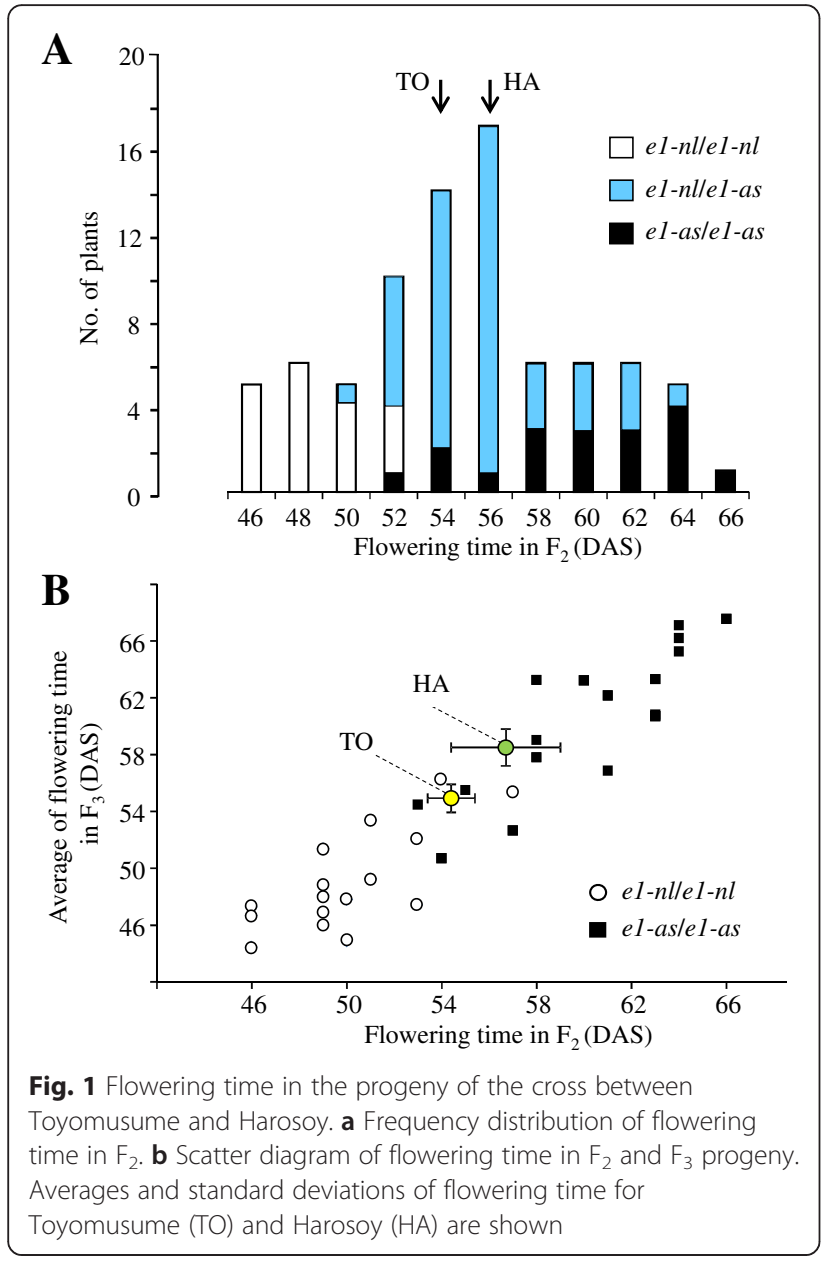

population. We determined the $E 1$ genotypes of $\mathrm{F}_{2}$ plants with an allele-specific DNA marker [29] and flanking simple sequence repeat (SSR) markers [15]. As expected, plants homozygous for $e 1-n l$ (from TO) flowered, on average, 11 days earlier than those homozygous for e1-as (from HA) (Fig. 1a). Since plants homozygous for each allele still varied considerably in flowering time, we carried out the progeny test for 16 plants homozygous for each allele. Flowering times of $F_{2}$ individuals were closely correlated with the average flowering times of their progeny (Fig. 1b). Parent-offspring correlation coefficients were 0.676 for the $e 1-n l$ homozygote and 0.823 for the e1-as homozygote, suggesting that a genetic factor(s) other than $E 1$ segregated in each of the two genotypic classes.

\section{Test for association between flowering time and SSR markers}

To detect flowering genes that segregated independently of $E 1$, we tested flowering time-SSR marker association in each of the $e 1-n l$ and $e 1$-as genotypic classes; we used 61 SSR markers located in the genomic regions where orthologs to Arabidopsis flowering genes are clustered [4]. Two markers were significantly associated with flowering time in $e 1-n l$ homozygotes and five in e1-as homozygotes (Table 1). Plants homozygous for the TO alleles (A) at all loci except Sat235 flowered later than those homozygous for the HA alleles (B). Only Sat_350 showed significant associations in both $e 1-n l$ and $e 1$-as genotypic classes. Sat_350 was located near the SSR marker Satt686 on LG J, which is a tagging marker for the $E 9$ gene identified in a cross between cultivated (TK780) and wild (Hidaka 4) soybeans [12]. Because TO is a parent of TK780 [44], which carries the recessive $e 9$ allele [12], it is plausible that the gene tagged by Sat_350 is identical to $E 9$ and that $\mathrm{TO}$ has the same recessive allele for late flowering as TK780.

\section{Fine-mapping and association analysis}

For fine-mapping of the E9 gene, a total of 300 seeds from two heterozygous $\mathrm{F}_{3}$ plants derived from the same $\mathrm{F}_{2}$ family (\#41) were genotyped for the SSR markers Sat_350 and BARCSOYSSR_16_1038. We detected eight recombinants (four progenies from each of two heterozygous $\mathrm{F}_{3}$ plants) in the flanking region, which were genotyped for seven additional SSR markers and three insertion/deletion (indel) markers (ID1, M5, and M7) used in the identification of $E 9$ [12]. The genotype at $E 9$ was estimated from the segregation pattern in the progeny test (Fig. 2a). Among the four plants derived from one $F_{3}$ parent, two plants (\#158 and \#175) flowered early and one (\#168) flowered late, whereas plant \#159 segregated for flowering time. Among the four plants derived from the other $F_{3}$ parent, two plants (\#262 and \#288) flowered early and one (\#276) flowered late, whereas one plant (\#281) segregated. By comparing the graphical genotypes and estimated $E 9$ genotypes, we delimited the QTL to a 40.1-kb region between markers BARCSOYSSR_16_1015 and BARCSOYSSR_16_1017, in which only the ID1 marker completely co-segregated with the genotype at $E 9$.

To confirm co-segregation between flowering time and ID1 genotype, we examined $14 \mathrm{~F}_{2}$ families homozygous for e1-nl and 14 homozygous for e1-as (Table 2). Among the $e 1-n l$ families, plants of two families homozygous for the TO allele flowered late, whereas plants of two families homozygous for the HA allele flowered early. A highly significant association between flowering time and marker genotypes was observed in the 10 heterozygous families. Similarly, a highly significant association was detected between flowering time and marker genotypes in the 5 heterozygous families with the e1-as genotype. Therefore, the variation in flowering time in each $F_{2}$ family could be mostly accounted for by the genotypes at the ID1 marker. 
Table 1 Association tests of SSR marker genotypes with flowering time

\begin{tabular}{|c|c|c|c|c|c|c|}
\hline \multirow[t]{2}{*}{ Marker } & \multirow[t]{2}{*}{ LG } & \multicolumn{3}{|c|}{ Average of flowering time (DAS) in } & \multicolumn{2}{|c|}{ One-way ANOVA } \\
\hline & & AA & $A B$ & BB & F value & Probability \\
\hline \multicolumn{7}{|c|}{ Plants homozygous for el-nl } \\
\hline Satt681 & $\mathrm{C} 2$ & 52.6 & 48.7 & 48.5 & 4.6 & 0.030 \\
\hline Sat_350 & J & 55.5 & 49.3 & 48.0 & 8.9 & 0.004 \\
\hline \multicolumn{7}{|c|}{ Plants homozygous for e1-as } \\
\hline Satt519 & B1 & 63.7 & 60.6 & 56.4 & 5.9 & 0.015 \\
\hline Sat_235 & $\mathrm{C} 1$ & 54.0 & 59.2 & 63.2 & 3.9 & 0.047 \\
\hline Satt031 & D2 & 63.3 & 60.4 & 56.0 & 5.0 & 0.025 \\
\hline Satt146 & $\mathrm{F}$ & 63.5 & 62.1 & 56.6 & 10.1 & 0.002 \\
\hline Sat_350 & J & 62.7 & 60.0 & 56.4 & 5.8 & 0.016 \\
\hline
\end{tabular}

16 plants homozygous for $e 1-n l$ and 16 plants homozygous for e1-aswere used in the association tests. A and B indicate the alleles from Toyomusume and Harosoy, respectively

LG, linkage group

\section{cDNA sequencing and expression analysis}

According to the Williams 82 reference genome sequence [45], the region delimited by fine mapping contained three genes: Glyma.16 g150700 (FT2a), Glyma.16 g150800 (EXOCYST COMPLEX PROTEIN EXO70), and Glyma.16 g150900 (TATD FAMILY DEOXYRIBONUCLEASE) (Fig. 2b). We focused on FT2a as a candidate for $E 9$ because of its importance in floral induction in soybean [22-28, 43]. cDNA sequence analysis was carried out for HA and TO, the Japanese cultivar Hayahikari (HY), and the parents (TK780 and Hidaka 4) of the recombinant inbred line (RIL) population used for the identification of $E 9$ [12]. There were no nucleotide substitutions in their coding regions, which were identical to that of Williams 82; a SNP (\#28; Additional file 1) after the stop codon was identified

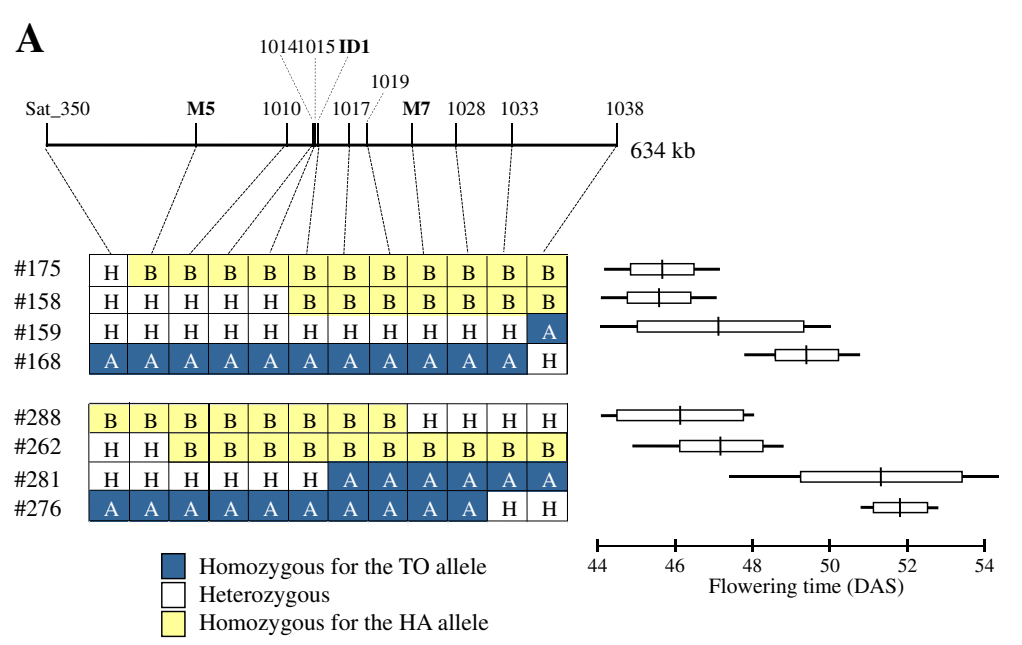

B

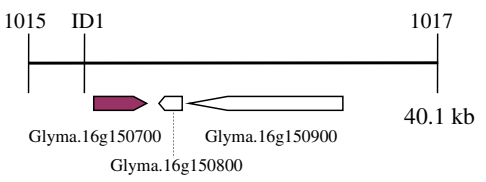

Glyma.16g150700; FT2a

Glyma.16g 150800; EXOCYST COMPLEX PROTEIN EXO70

Glyma.16g150900; TATD FAMILY DEOXYRIBONUCLEASE

Fig. 2 Fine mapping of the $E 9$ locus and annotated genes in the delimited genomic region. a Eight recombinants (four from each of two $F_{3}$ heterozygous plants) in the region between Sat_350 and BARCSOYSSR_16_1038 were genotyped at 7 BARCSOYSSR (1010 to 1033) and 3 indel markers (bold). The genotype at E9 was estimated by progeny testing. The ranges (horizontal lines), averages (vertical lines), and standard deviations (open boxes) of flowering time (DAS: days after sowing) are indicated. b Three annotated genes in a delimited genomic region 
Table 2 Association tests of ID1, a tagging marker of E9, with flowering time

\begin{tabular}{|c|c|c|c|c|c|}
\hline \multirow{2}{*}{$\begin{array}{l}\mathrm{F}_{2} \\
\text { Plant number }\end{array}$} & \multicolumn{3}{|c|}{ Average (SD) of flowering time (DAS) in $F_{3}$} & \multicolumn{2}{|c|}{ One-way ANOVA } \\
\hline & $\mathrm{AA}$ & $A B$ & $\mathrm{BB}$ & F value & Probability $\left(10^{-3}\right.$ \\
\hline \multicolumn{6}{|c|}{$F_{2}$ families with el-nl/el-nl } \\
\hline \#34 & & & $44.5(1.1)$ & & \\
\hline \#66 & & & $44.9(0.8)$ & & \\
\hline \#02 & $51.8(2.2)$ & $44.6(1.4)$ & $44.3(0.5)$ & 27.2 & 0.005 \\
\hline \#05 & $53.0(1.7)$ & $46.3(1.4)$ & $43.0(0.8)$ & 45.6 & 0.000 \\
\hline \#25 & $52.8(2.8)$ & $47.7(1.9)$ & $44.8(1.2)$ & 22.1 & 0.018 \\
\hline \#27 & $52.6(2.1)$ & $45.5(0.8)$ & $44.0(0.0)$ & 73.2 & 0.000 \\
\hline \#28 & $56.0(1.7)$ & $52.1(1.2)$ & $49.5(2.4)$ & 14.4 & 0.223 \\
\hline \#41 & $54.0(2.0)$ & $46.2(1.7)$ & $44.2(1.6)$ & 40.7 & 0.000 \\
\hline \#50 & $56.0(1.0)$ & $53.6(2.1)$ & $50.4(2.9)$ & 8.8 & 2.324 \\
\hline \#79 & $56.0(1.4)$ & $47.8(1.8)$ & $45.4(1.3)$ & 61.3 & 0.000 \\
\hline \#81 & $55.3(1.6)$ & $46.3(1.0)$ & $44.7(0.5)$ & 156.8 & 0.000 \\
\hline \#82 & $56.4(0.5)$ & $52.1(1.0)$ & $46.9(3.7)$ & 26.2 & 0.006 \\
\hline \#18 & $55.4(1.5)$ & & & & \\
\hline \#46 & $56.4(1.5)$ & & & & \\
\hline \multicolumn{6}{|c|}{$\mathrm{F}_{2}$ families with e1-as/e1-as } \\
\hline$\# 12$ & & & $60.7(1.9)$ & & \\
\hline \#29 & & & $50.7(1.5)$ & & \\
\hline \#30 & & & $52.7(2.9)$ & & \\
\hline \#43 & & & $54.5(2.4)$ & & \\
\hline \#22 & $64.8(1.3)$ & $57.5(1.6)$ & $52.0(2.8)$ & 92.0 & 0.000 \\
\hline \#36 & $65.7(0.6)$ & $57.3(3.2)$ & $50.4(3.8)$ & 57.9 & 0.020 \\
\hline \#48 & $65.7(1.6)$ & $59.5(2.7)$ & $55.0(1.7)$ & 63.7 & 0.005 \\
\hline \#69 & $66.7(1.4)$ & $62.5(3.8)$ & $59.3(2.1)$ & 42.6 & 7.615 \\
\hline \#73 & $65.4(1.5)$ & $55.6(2.7)$ & $55.0(4.0)$ & 58.9 & 0.015 \\
\hline \#13 & $66.2(0.8)$ & & & & \\
\hline \#16 & $67.4(0.6)$ & & & & \\
\hline \#33 & $63.2(3.2)$ & & & & \\
\hline \#76 & $63.3(1.9)$ & & & & \\
\hline \#78 & $65.9(0.8)$ & & & & \\
\hline
\end{tabular}

The progeny of 14 plants homozygous for $e 1-n l$ and 14 plants homozygous for $e 1-a s$ were used in the association tests. A and B indicate the alleles from Toyomusume and Harosoy, respectively

between HA and TO or HY. We then compared the expression profiles of FT2 $a$ under short day (SD) and long day (LD) conditions in plants homozygous for the TO allele and those homozygous for the HA allele at ID1 in the progeny of $10 \mathrm{~F}_{2}$ families with the e1-nl/e1-nl genotype that segregated for E9. The FT2a transcript abundance was analyzed at Zeitgeber time 3. In all tested families, plants with the HA allele had higher FT2a expression than plants with the TO allele, regardless of daylength, although the expression was much higher in SD than LD in both homozygotes (Fig. 3). The lower expression of FT2 $a$ in plants with the TO allele was further confirmed in the diurnal expression patterns in TO and
HA: the expression levels of TO were very low across any sampling times compared with that of HA (Additional file 2). Thus, late flowering in plants homozygous for the TO allele at ID1 was tightly associated with reduced $F T 2 a$ expression.

\section{Sequence analysis of the FT2a genomic region}

In Arabidopsis, FT is regulated by various transcription factors, which bind to the promoter or to the first intron and 3 ' downstream region $[1,3]$. To detect the cause of the reduced FT2 $a$ expression, we first sequenced the $5^{\prime}$ upstream region of $F T 2 a$ in the three cultivars and in TK780 and Hidaka 4. We detected 8 SNPs and 6 indels. 

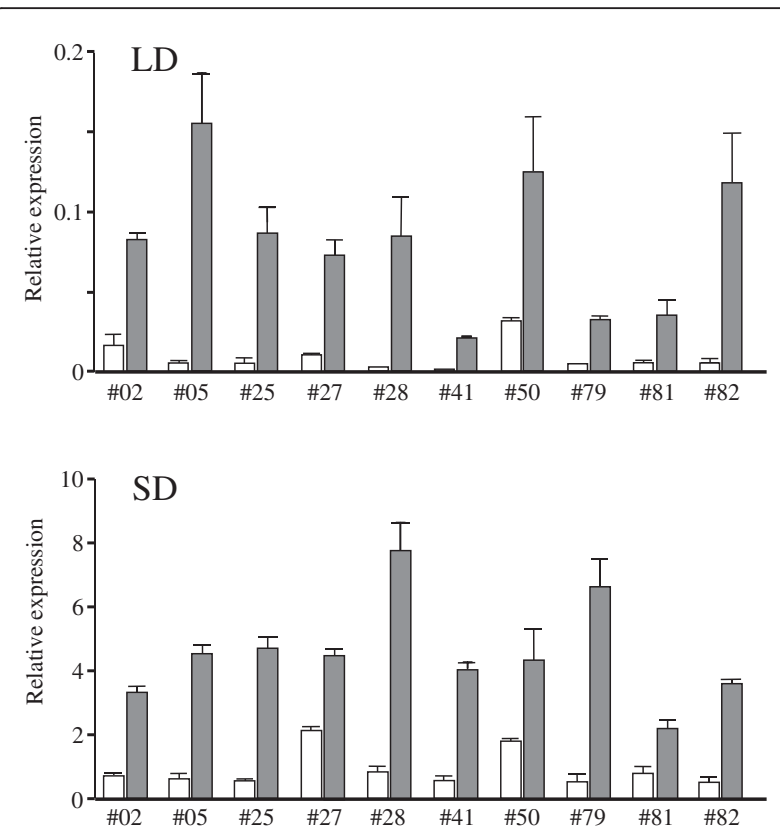

Fig. 3 FT2 $a$ expression in the progeny of $F_{2}$ plants from a cross between Toyomusume and Harosoy. Four plants from the progeny of each $F_{2}$ plant, which were homozygous for the Toyomusume allele (white bars) or the Harosoy allele (gray bars) at the ID1 tagging marker for FT2a, were used. Relative mRNA levels are expressed as the ratios to $\beta$-tubulin transcript levels

The sequences of TO and TK780 were identical to each other, but differed from those of HA and Hidaka 4 in a 43-bp indel in the promoter and a 10-bp indel in the $5^{\prime}$ UTR, which were located 731 and 47 bp upstream of the start codon, respectively, and in two SNPs (\#2 and \#4) (Additional file 1). The sequence of $\mathrm{HY}$ was similar to those of TO and TK780 (including the 43-bp segment), but differed from them in one SNP (\#1), a 4-bp indel 274 bp upstream of the start codon, and the 10-bp indel in the $5^{\prime}$ UTR.

We also sequenced the introns and the 3 '-downstream region in TO, HA, and $\mathrm{HY}$ to test whether the polymorphism(s) observed in the promoter and 5' UTR could be responsible for late flowering in TO. The primers based on the gene model Glyma.16 g150700 worked well for PCR amplification of these regions except for the first intron of TO. To sequence the first intron in TO, we used genome walking. Nested PCR analysis of genomic libraries produced an amplicon of $370 \mathrm{bp}$ from the library constructed by using EcoRV. Sequencing revealed that it consisted of an unknown sequence of 137-bp fused with a 233-bp segment of the first intron of FT2a proximal to the second exon. A BLAST search of the NCBI genome database showed that the former sequence was identical to a part of an LTR of SORE-1 (AB370254), which has been previously detected in a recessive allele at the E4 locus [21, 46]. The inserted retrotransposon and its flanking regions were then amplified by nested PCR and sequenced. The retrotransposon was 6,224 bp long; its sequence was $100 \%$ identical to the LTRs of SORE-1 and $99.7 \%$ identical to its coding region. Using a DNA marker for the SORE-1 detection, we confirmed that TK780 also had SORE-1 in the first intron, but Hidaka 4, HA, and HY did not. We detected a total of 17 polymorphisms (10 SNPs, 2 indels, and 5 SSRs) from the first intron to 3' downstream regions among the three cultivars (Additional file 1).

Thus, three early-maturing cultivars-TO, HA, and $\mathrm{HY}$-had different FT2 $a$ sequences, which were designated as the FT2a-TO, FT2a-HA, and FT2a-HY alleles. FT2 $a-T O$ differed from both FT2a-HA and FT2a-HY in the 10-bp deletion in the 5' UTR, and in SNP \#17 and the SORE-1 insertion in intron 1 (Fig. 4a, Additional file $1)$. By using the database of plant cis-acting regulatory DNA elements (PLACE) [47], we detected a W-box element (AGTCAAA) that was created by SNP \#17 in TO, and two cis-elements, RBCSCONSENSUS (AATCCAA) and ARR1AT (NGATT), in the genomic region flanking the SORE-1 integration site.

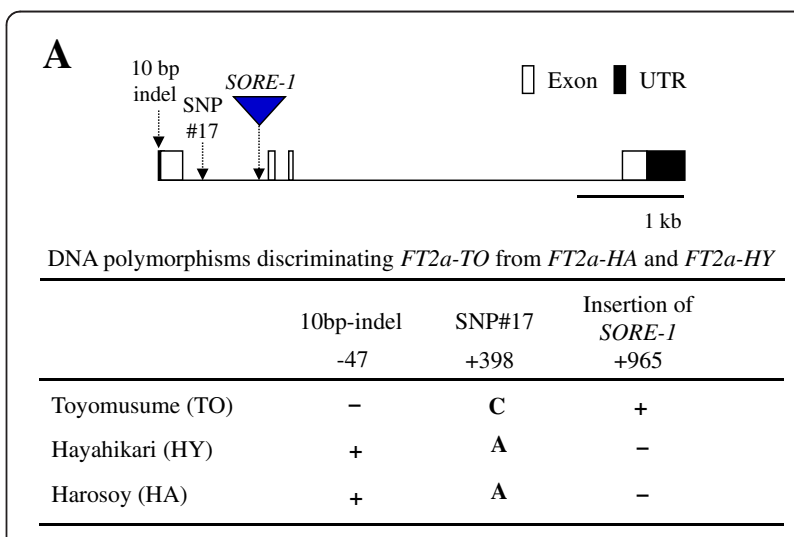

B

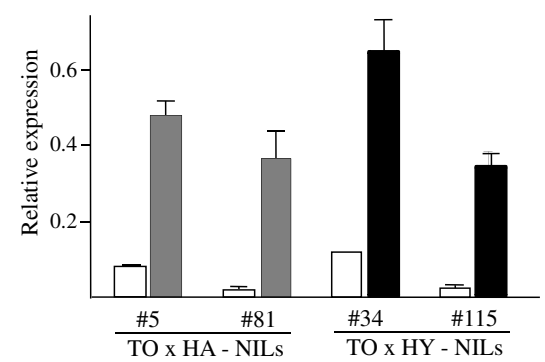

Fig. 4 DNA polymorphisms that discriminate between the FT2a alleles and FT2a transcript abundance in their NILs. a Genomic positions and types of three DNA polymorphisms between Toyomusume (TO) and both Harosoy (HA) and Hayahikari ( $\mathrm{HY}$ ) b FT2a expression in 20-DAS-old plants of NILs for FT2a-TO (white) and FT2a-HA (gray) or FT2a-HY (black) under SD conditions. Relative mRNA levels are expressed as the ratios to $\beta$-tubulin transcript levels 
Expression of different FT2a alleles in near-isogenic lines and photoperiod-insensitive accessions

We developed four sets of NILs for the above three FT2 $a$ alleles from the progeny of $\mathrm{F}_{5}$ heterozygous plants: two from the cross between TO and HA (\#5 and \#81) and two from the cross between TO and HY (\#34 and \#115). We found that, under SD conditions, FT2a-TO expression was much lower than that of FT2a-HA and FT2a-HY (Fig. 4b).

Using 3 markers, we selected five photoperiodinsensitive e3 e4 cultivars, all of which had the 10-bp deletion in 5' UTR, but differed in SNP \#17 and in the presence or absence of SORE-1 (Fig. 5a). We analyzed FT2 $a$ expression in fully-expanded trifoliate leaves at different leaf stages (first, second, and third true leaves) (Fig. 5b). FT2a expression was markedly low in all stages in Karafuto 1, but was relatively high in the other four. Because Karafuto 1 differed from the other cultivars only in the presence of SORE-1, low expression of FT2a-TO was caused by the insertion of SORE-1, not by the 10-bp deletion or by SNP \#17.
RNA processing and DNA methylation at the FT2a locus Transposable elements (TEs) in introns often affect chromatin structure and modify RNA processing of the host gene and, therefore, influence its expression patterns [48-50]. Using qRT-PCR on cDNA synthesized with random primers, which targeted different regions, we analyzed FT2 $a$ expression in two sets of NILs for $F T 2 a-T O$ and FT2a-HY grown in SD. In all three targeted regions (a-c in Fig. 6a), the FT2a transcript abundance was considerably lower $(1 / 5$ to $<1 / 10)$ in NILs for FT2a-TO than in NILs for FT2a-HY (Fig. 6b).

To analyze FT2 $a$ RNA processing in FT2a-TO, we performed semi-quantitative RT-PCR on cDNAs synthesized with random primers. No amplicon was detected in regions a (from exon 1 to intron 1), b and $\mathrm{c}$ (from exon 1 to $S O R E-1$ ), or d and e (from SORE-1 to exon 2), although the expected amplicons were observed in PCR on genomic DNA of the NIL for FT2a-TO (Fig. 7). For region $\mathrm{f}$ (from exon 1 to exon 2 ), a fragment $(\sim 150 \mathrm{bp})$ was amplified in both NILs, although signal intensity was much higher in the NIL for FT2a-HY than in the

\section{$\mathbf{A}$}

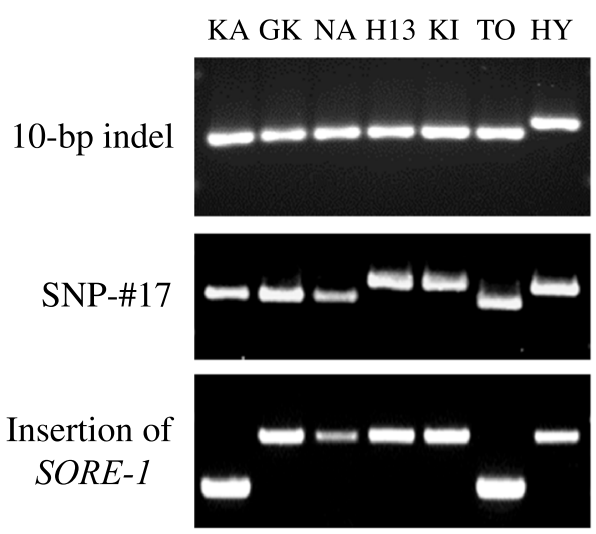

B

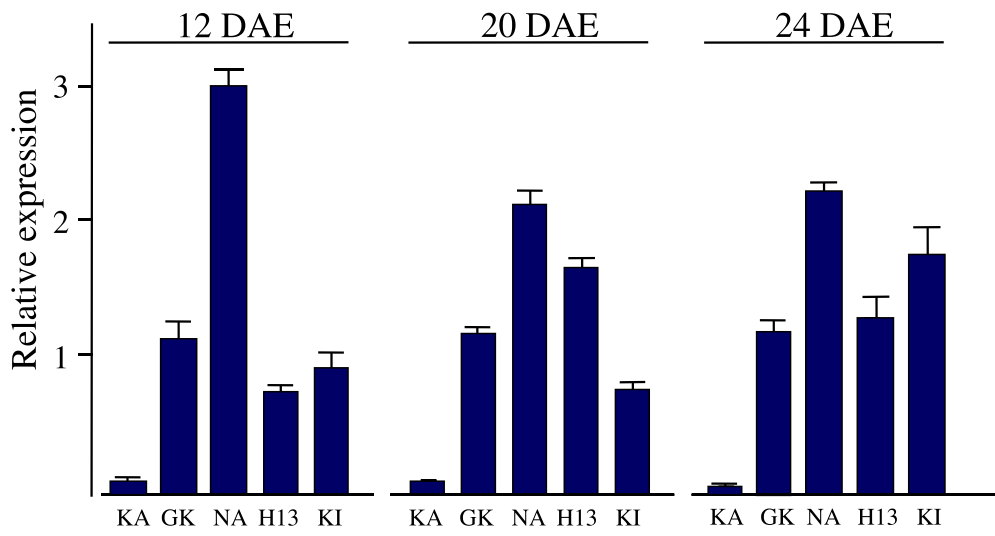

Fig. 5 FT2a transcript abundance in photoperiod-insensitive e3 e4 cultivars under SD conditions. a DNA polymorphisms in the 10-bp indel, SNP \#17, and SORE-1 insertion. b FT2a expression at the first (12 days after emergence: DAE), second (20 DAE), and third (24 DAE) leaf stages. Relative mRNA levels are expressed as the ratios to $\beta$-tubulin transcript levels. KA, Karafuto 1; GK, Gokuwase-Kamishunbetsu; NA, Napoli; H13, Heihe 13; KI, Kitamusume; TO, Toyomusume; HY, Hayahikari 
A

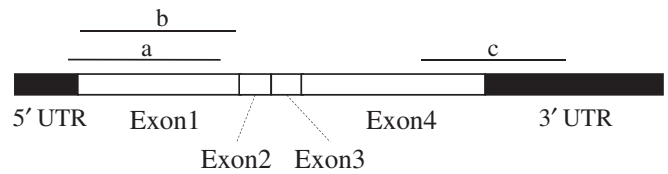

B
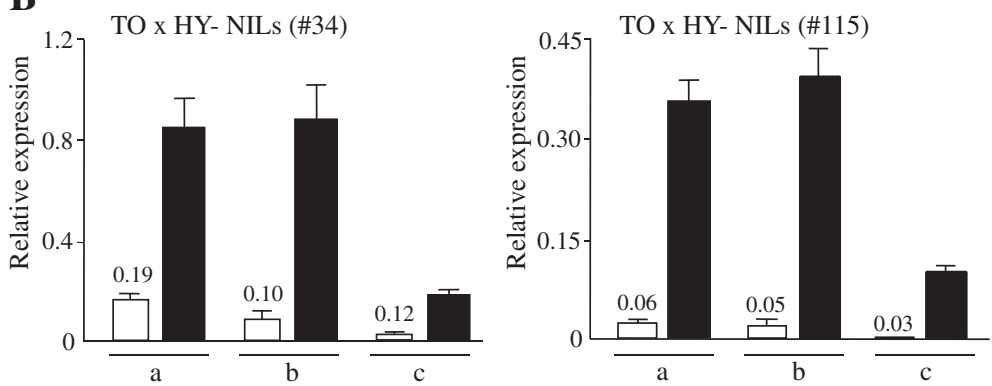

Fig. 6 FT2a transcript abundance in two sets of NILs for FT2a-TO and FT2a-HY alleles. a Three regions (a-c) in the FT2a coding region used to assess transcript abundance. The 5' UTR and 3' UTR are a part of exon 1 and exon 4, respectively. b FT2a expression analyzed in 20-DAS plants under SD conditions. Relative mRNA levels are expressed as the ratios to $\beta$-tubulin transcript levels. cDNA was synthesized with random primers. Numbers above the white bars are the ratios of the expression levels in NILs for FT2a-TO (white bars) to those in FT2a-HY (Black bars)

NIL for FT2a-TO; as expected, genomic PCR produced fragments of 7,293 bp in the NIL for FT2a-TO and 1,064 bp in the NIL for FT2a-HY (Fig. 7b). These results suggest that intron 1 with the SORE-1 insertion could be spliced out in the NIL for FT2a-TO.

Next, we examined FT2a expression in heterozygous siblings of NILs; this analysis was based on the fact that SNP \#28 after the stop codon (Additional file 1) created a DdeI restriction site in FT2a-HA, but not in FT2a-TO and FT2a-HY. By performing RT-PCR and digesting the product with $D d e I$, expression of $F T 2 a-T O$ can be distinguished from that of $F T 2 a-H A$ in heterozygous plants. In the NILs-\#5 for FT2a-TO and FT2a-HA, and its siblings, the $F T 2 a$ transcript level was high in homozygotes for $F T 2 a-H A$, slightly lower in heterozygotes, and very low in homozygotes for FT2a-TO (Fig. 8a). Digestion of PCR products revealed that in heterozygotes, the transcript level of FT2a-HA was much higher than that of FT2a-TO. This difference suggests that the lower expression of FT2a-TO was caused by allele-specific transcriptional repression rather than sequence-specific RNA degradation of RNA silencing that decreases the levels of transcripts from both alleles.

We also evaluated the methylation of FT2a-TO and FT2a-HY. Methylation-dependent McrBC restriction digestions and mock digestions of genomic DNA were used to analyze cytosine methylation in NILs for FT2a$T O$ and FT2a-HY. Semi-quantitative PCR was performed using primers designed for each of the targeted regions to be singly amplified (Fig. 8b). There was no difference in PCR amplification of genomic regions $\mathrm{a}-\mathrm{f}$ and $\mathrm{h}-\mathrm{k}$ in the McrBC-digested and mock-digested samples in both NILs (Fig. 8c). In contrast, no amplicons were detected for regions S1-S3 (which include the LTRs of SORE-1 and FT2a regions flanking the LTRs) after $M c r B C$ digestion in the NIL for FT2a-TO, although fragments of expected sizes were amplified from mockdigested DNA. PCR on both $M c r B C$-digested and mockdigested DNAs produced the expected amplicons in region S4 (which did not include the LTR sequence) of the NIL for FT2a-TO and in genomic region $g$ (which did not contain SORE-1) of the NIL for FT2a-HY. Taken together, these data indicate that SORE-1 was highly methylated, but methylation appeared not to extend to the FT2a genomic region flanking SORE-1. The same result was obtained for plants grown in LD (data not shown), which indicates that lower mRNA level of FT2a-TO is associated with SORE-1 methylation in both SD and LD conditions.

\section{Discussion}

\section{Maturity gene E9 is FT2a}

Flowering time in the $\mathrm{F}_{2}$ and $\mathrm{F}_{3}$ progeny of a cross between TO and HA co-segregated with the alleles at the $E 1$ and $E 9$ loci. Fine mapping delimited $E 9$ to a $40.1-\mathrm{kb}$ region that contained three genes, including $F T 2 a$, a soybean ortholog of $F T$ (Fig. 2). Sequencing and expression analysis suggested that $F T 2 a$ is the most likely candidate for $E 9$, and delayed flowering due to $e 9$ is most likely caused by the reduced $F T 2 a$ transcript abundance. Despite sequence identity in the coding regions, we detected several SNPs and indels of 4-43 bp in the promoter and 5' UTR among cultivars and accessions tested; this is consistent with a previous report [42]. 

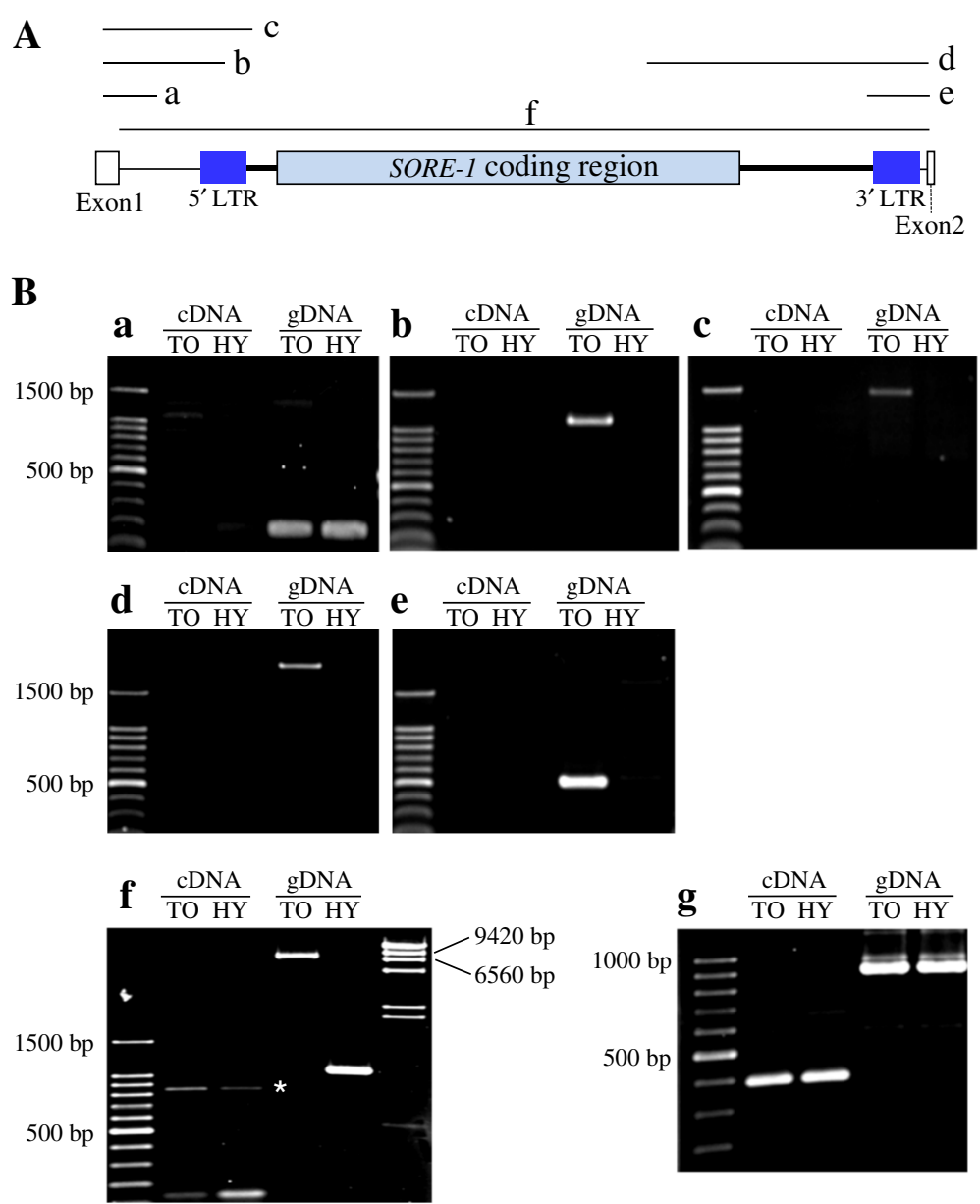

Fig. 7 FT2a RNA processing in the first intron with SORE-1 insertion. a a-f, Regions examined. b Semi-quantitative PCR analysis of FT2a expression in NILs (\#115) for FT2a-TO (TO) and FT2a-HY (HY) in 20-DAS plants under SD conditions. CDNA was synthesized with random primers. $g$, amplification of the $\beta$-tubulin transcript. *, nonspecific amplification

However, expression analysis of NILs and photoperiodinsensitive accessions carrying different FT2 $a$ alleles revealed that the polymorphisms in the promoter and $5^{\prime}$ UTR were not responsible for different FT2a expression levels (Figs. 4 and 5). TO also differed from HA and HY by a SNP and a SORE-1 insertion in the first intron, of which the latter was solely associated with the FT2a expression levels (Fig. 5). Thus, our study reveals that the insertion of SORE-1 attenuated FT2a expression and delayed flowering. The soybean genome possesses a total of ten $F T$ orthologs, among which six retain the $F T$ function and can promote flowering of Arabidopsis $f t$ mutants $[22,23]$ or Col-0 [24, 25]. All of the six homologs could therefore function as potential floral inducers in soybean, although only two of them, FT2a and FT5a, have been extensively characterized in studies of molecular mechanisms of flowering $[15,19,22,24,26-28,40,43]$. This study demonstrates that different levels of FT2a expression directly regulate natural variation in flowering time in soybean.
Factors responsible for attenuation of $F T 2 a$ expression Plant TEs inserted in introns may affect RNA processing $[48,49]$ and render their host genes susceptible to short interfering RNA (siRNA)-mediated silencing [50]. Our results show that the first intron (including SORE-1) is spliced out, because no primary RNA transcripts that would cover FT2a exons and SORE-1 were detected while the spliced products were detected (Fig. 7). Thus, SORE-1 insertion did not markedly interfere with FT2a RNA processing.

We found that the reduction in FT2a-TO transcript abundance was caused by allele-specific transcriptional repression due to the insertion of SORE-1, the LTRs and adjacent sequences of which were highly methylated (Fig. 8). Therefore, epigenetic mechanisms likely account for the reduction in FT2a-TO transcript levels. RNAdirected DNA methylation or the resulting chromatin modifications regulate gene expression by interfering with transcription factor binding, leading to different expression profiles for different transcription factors 
A
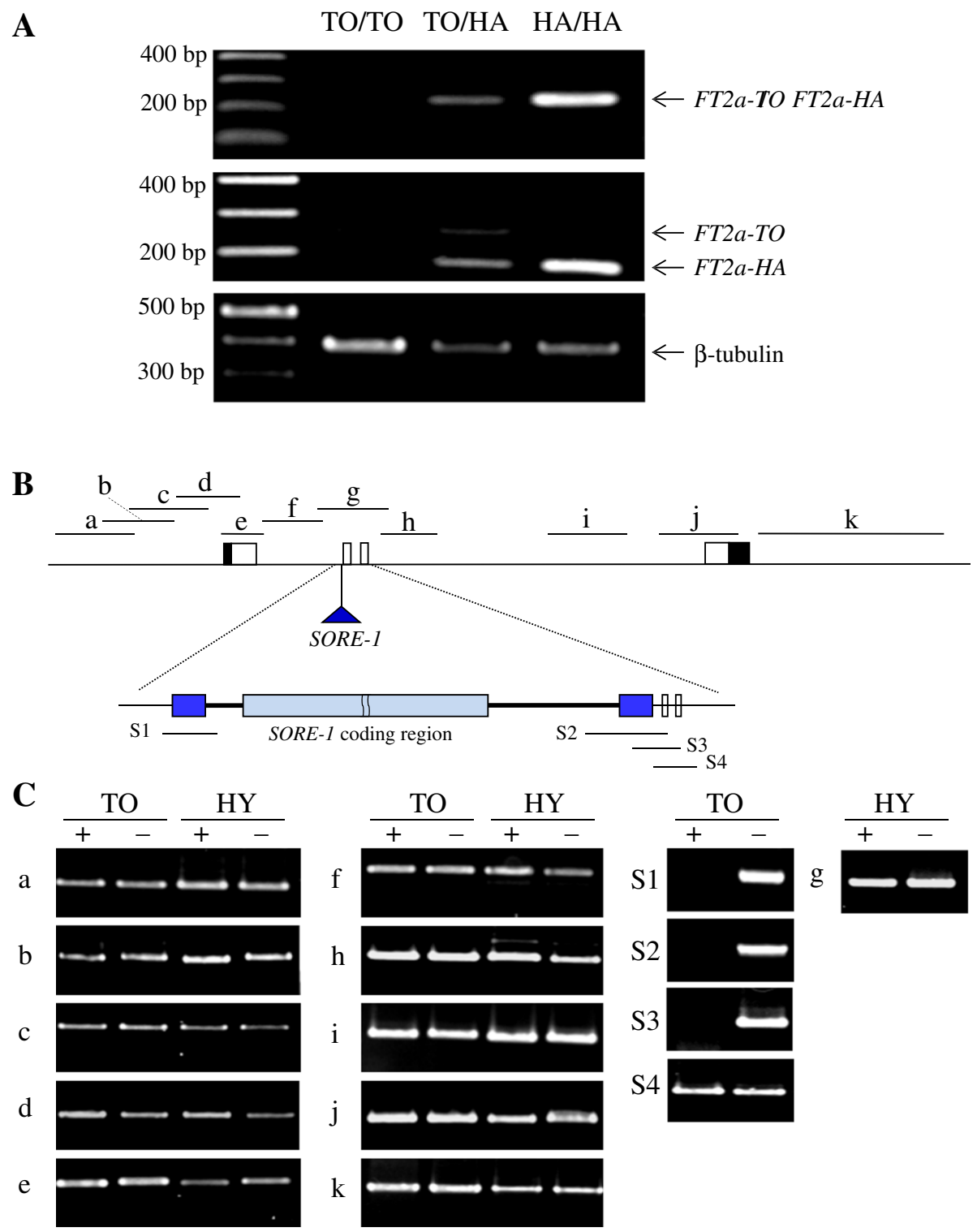

Fig. 8 Transcript abundance of different alleles and DNA methylation in the FT2a genomic region. a Transcript abundance of different FT2a alleles assayed by allele-specific restriction digestion. $\mathbf{b}$ Diagram of the FT2a genomic region showing the position of SORE-1 insertion. Amplicons were analyzed by semi-quantitative PCR after McrBC or mock digestion; the amplified regions are designated as a to $k$ and S1 to S4. Exons, white; UTRs, black; LTRs of SORE-1, gray. c Genomic DNA from leaves of 20-day-old plants of NILs for FT2a-TO (TO) and FT2a-HY (HY) grown under SD conditions was digested with McrBC (+) or mock-digested (-) and amplified by PCR. Amplicons were visualized in agarose gels

[50-52]. PLACE analysis detected two cis-elements, RBCSCONSENSUS and ARR1AT, in the region flanking the SORE-1 integration site in the first intron. However, the functions of the two elements in FT2a expression are unclear. A further test is thus needed to determine the functions of the two cis-elements or nearby unknown elements in the regulation of FT2a expression and whether SORE-1 insertion interrupts binding of a transcriptional factor(s) to these cis-elements.

Methylation-mediated gene repression by intronic TEs is well characterized in Arabidopsis FLOWERING
LOCUS C (FLC), which encodes a transcription factor containing a MADS domain that inhibits FT expression $[53,54]$. In Col-0, the functional FLC allele is highly expressed in the presence of FRIGIDA and causes extremely late flowering [54]. In contrast, in ecotype Landsberg erecta (Ler), the FLC allele has a 1,224-bp non-autonomous Mutator-like TE in intron 1 and is expressed at low levels due to its transcriptional silencing through histone H3-K9 methylation, which is triggered by siRNA generated from homologous TEs [50]. FLC-Ler, however, can still be regulated by genes in 
the autonomous flowering pathway and by genes involved in vernalization, because the TE insertion does not affect the transcription factor-binding sites in intron 1 [50]. Similarly to the FLC-Ler allele, the expression of FT2a-TO is repressed due to epigenetic modification caused by the insertion of SORE-1 in intron 1 . However, FT2a-TO expression was still higher in SD than in LD (Fig. 3). Virus-induced silencing of E1-like genes (repressors of $F T 2 a$ and $F T 5 a$ ) lowers photoperiod sensitivity of TO by up-regulating the expression of both $F T 2 a$ and FT5a [28]. The regulation of FT2a expression by E1-like and other genes involved in photoperiod responses is thus retained in FT2a-TO plants. The FT2a-TO allele may thus be involved in flowering as a leaky allele, not a dysfunctional allele.

\section{Origin and adaptive role of the $e 9$ allele}

SORE-1 was first detected in a recessive allele at the E4 locus encoding phytochrome $\mathrm{A}$; its insertion in the first exon caused a premature stop codon and resulted in a dysfunctional truncated protein [21]. DNA marker analysis revealed that the $e 4$ allele with the SORE-1 insertion is present mainly in landraces from northern Japan [46], although it has been used in breeding of photoperiodinsensitive cultivars in high-latitude regions of other countries [29]. This insertion in the E4 gene may thus have played an adaptive role in expanding the areas of soybean cultivation to higher latitudes. Our preliminary survey of the insertion of SORE- 1 in the FT2a allele suggests that FT2a-TO is a region-specific allele detected in only a few local varieties established in Sakhalin and northern Hokkaido among photoperiod-insensitive landraces and cultivars having the $e 4$ allele with the SORE-1 insertion. Therefore, the insertion of SORE-1 in the first intron of FT2a-TO may be of recent origin.

Our preliminary survey also suggests that the cultivar Toshidai-7910, introduced from Sakhalin with Karafuto 1, was the source of the FT2 $a$ allele with the SORE-1 insertion in TO [44]. Similar to TO, both Toshidai-7910 and Karafuto 1 have a null allele at the $E 1$ locus, but, unlike TO, they have recessive alleles at E3 and E4 ([18] and this study). This is a maturity genotype that permits extremely early flowering and maturation and enables seed production in cold climates with a limited frost-free season. Because FT2 $a$ and FT5a control flowering redundantly [24, 27], the $e 9(F T 2 a-T O)$ allele could have been selected in the presence of functional FT5 $a$ because it maintains vegetative growth. It is thus another example of the adaptive role of SORE-1 insertion as indicated by Kanazawa et al. [46]. The $e 9$ allele may also be useful for developing cultivars adapted to a shorter photoperiod in low-latitude environments where flowering is strongly promoted. In such environments, a longer vegetative phase, a so-called long-juvenile trait, is desirable. A leaky allele similar to $e 9$ may be useful for reducing the transcript levels of FT2 $a$ under SD conditions, in addition to long-juvenile genes reported so far, such as E6 [9] and $j$ [13]. A further study is needed to evaluate the adaptive significance of $e 9$ under $\mathrm{SD}$ conditions.

\section{Conclusions}

The present study revealed that the soybean maturity gene $E 9$ is FT2a, an ortholog of Arabidopsis FT, and that its recessive allele delays flowering through lower transcript abundance. FT2a is thus directly involved in the natural variation in flowering time in soybean. The attenuation of FT2a expression is caused by allele-specific transcriptional repression caused by the insertion of $S O R E-1$ in the first intron. The recessive $e 9$ allele is a leaky allele; its regulation by other genes involved in photoperiod response is retained. It may thus maintain vegetative growth in early-flowering genetic backgrounds, and also be useful as a long-juvenile allele in cultivar development in low-latitude regions, where flowering is strongly promoted.

\section{Methods}

\section{Plant materials}

Three early-maturing soybean cultivars were used in this study: the Canadian cultivar Harosoy (L58-266; HA) and two Japanese cultivars, Toyomusume (TO) and Hayahikari (HY). HA and TO have the e2/e2, E3/ $E 3$, and E4/E4 allele composition; HA has a hypomorphic e1-as allele, whereas TO lacks the genomic region $(\sim 130 \mathrm{~kb})$ containing the entire $E 1$ gene $(e 1-n l$ allele) [18]. HY has the E1/E1,e2/e2,e3/e3, and e4/e4 allelic composition and is photoperiod-insensitive [18]. We developed four sets of $F_{6}$ near-isogenic lines (NILs) for the $E 9$ gene from the progeny of $\mathrm{F}_{5}$ heterozygous plants: two sets from the cross between $\mathrm{TO}$ and HA and two from the cross between TO and HY. All NILs had the same genotype (e1-nl/e1-nl,e2/ $e 2, E 3 / E 3$, and $E 4 / E 4)$ as TO. The breeding line TK780 and the wild soybean accession Hidaka 4 were used for sequencing because they were parents of the recombinant inbred population used for identification of the $E 9$ gene [12, 14]. Five photoperiod-insensitive accessions (Karafuto 1, Gokuwase-Kamishunbetsu, Nawiko, Heihe 13, and Kitamusume) were used for expression analyses.

\section{Segregation analysis and fine mapping of the $E 9$ gene}

Seeds of the $F_{2}$ population $(n=82)$ and both parents, TO and HA $(n=10)$, were sown in paper pots on 25 May 2012, and 10 days later seedlings were transplanted into soil at an experimental farm of Hokkaido University, Sapporo $\left(43^{\circ} 07^{\prime} \mathrm{N}, 141^{\circ} 35^{\prime} \mathrm{E}\right)$. The $82 \mathrm{~F}_{2}$ plants were 
genotyped with a DNA marker at the $E 1$ locus [18] and its flanking SSR marker [15], and 16 plants homozygous for $e 1-n l$ and 16 plants homozygous for e1-as were selected for the progeny test. Seeds of each $F_{2}$ plant were sown on 25 May 2013, and 10 days later 20 seedlings were transplanted into the same field. The number of days from sowing to the first flower opening (R1) [55] of each plant was recorded. For fine-mapping of the $E 9$ gene, a total of 300 seeds from two heterozygous $F_{3}$ plants derived from the same $F_{2}$ family (\#41) were genotyped for SSR and indel markers flanking this gene. Eight recombinants between markers were cultivated in a glasshouse during winter, and the seeds produced were used for the progeny test during summer (sowing date: 15 May 2014, $n=20$ ).

\section{DNA marker analysis}

Total DNA was extracted from trifoliate leaves as described [56] and from seeds as described [15]. Sixty-one SSR markers mapped on the consensus map (SOYBASE; http://soybase.org/sbt/) $[57,58]$ and located in the genomic regions where orthologs to Arabidopsis flowering genes are clustered [4] were chosen for tests of their association with flowering time. Data analysis in association tests were performed by one-way analysis of variance. The following DNA markers flanking $E 9$ were used for fine mapping: nine SSR markers available in the genomic sequence database of Williams 82 (Gmax v. 2.0; http://www.phytozome.net/soybean) [44] and three indel markers [12]. Each PCR contained $30 \mathrm{ng}$ of total genomic DNA as template, $1 \mu \mathrm{l}$ of each primer $(10 \mu \mathrm{M})$ and dNTP $(2.5 \mathrm{mM}), 0.5 \mu \mathrm{l}$ of ExTaq polymerase, and $2.5 \mu \mathrm{l}$ of 10x ExTaq buffer (Takara, Otsu, Japan) in a total volume of $25 \mu \mathrm{l}$; amplification conditions were 35 cycles at $94{ }^{\circ} \mathrm{C}$ for $30 \mathrm{~s}, 56{ }^{\circ} \mathrm{C}$ to $60{ }^{\circ} \mathrm{C}$ (depending on the primers used) for $30 \mathrm{~s}$, and $72{ }^{\circ} \mathrm{C}$ for $30-90 \mathrm{~s}$. PCR products were separated by electrophoresis in $10.5 \%(\mathrm{w} / \mathrm{v})$ polyacrylamide gels (for SSR markers) or $1 \%$ agarose gels (for indels), stained with ethidium bromide, and visualized under UV light.

\section{Expression analysis}

Plants were grown in growth cabinets under SD (12 h) or LD $(18 \mathrm{~h})$ conditions at $24{ }^{\circ} \mathrm{C}$. Fully developed trifoliate leaves of four 20-day-old plants were sampled as a bulk at Zeitgeber time 3 to compare the expression levels of FT2a between plants with different alleles or every three hours to determine the diurnal patterns in TO and HA. Leaves were immediately frozen in liquid $\mathrm{N}_{2}$, and stored at $-80{ }^{\circ} \mathrm{C}$. Total RNA was isolated from frozen tissues by lithium chloride precipitation according to Napoli et al. [59], except that DNase I (Takara Bio, Otsu, Japan) was used to remove genomic DNA. cDNA was synthesized from $1 \mu \mathrm{g}$ of total RNA using an oligo
(dT) 18 primer or random primer cocktail (Takara Bio, Otsu, Japan) according to Dwiyanti et al. [60]. FT2a transcript levels were determined by semi-quantitative RT-PCR or quantitative real-time PCR (qRT-PCR). Each of semi-quantitative RT-PCR contained $0.5 \mu \mathrm{g}$ of cDNA $(0.1 \mu \mathrm{g}$ for total genomic DNA used as a control) as template, $1 \mu \mathrm{l}$ of each primer $(10 \mu \mathrm{M})$ and dNTP $(2.5 \mathrm{mM}), 0.5 \mu \mathrm{l}$ of ExTaq polymerase, and $2.5 \mu \mathrm{l}$ of $10 \times$ ExTaq buffer in a total volume of $25 \mu \mathrm{l}$; amplification conditions were 33 cycles at $94{ }^{\circ} \mathrm{C}$ for $30 \mathrm{~s}, 60^{\circ} \mathrm{C}$ or $64{ }^{\circ} \mathrm{C}$ for $30 \mathrm{~s}$ (depending on the primers used), and $72{ }^{\circ} \mathrm{C}$ for $30 \mathrm{~s}-$ $8 \mathrm{~m}$ (depending on the sizes of amplified fragments). The qRT-PCR mixture $(20 \mu \mathrm{L})$ contained $0.05 \mu \mathrm{L}$ of the cDNA synthesis reaction, $5 \mu \mathrm{L}$ of $1.2 \mu \mathrm{M}$ primer premix, and $10 \mu \mathrm{L}$ SYBR Premix ExTaq Perfect Real Time (Takara, Otsu, Japan). A CFX96 Real-Time System (Bio-Rad Laboratories Japan, Tokyo, Japan) was used. The PCR cycling conditions were $95^{\circ} \mathrm{C}$ for 3 min followed by 35 cycles of $95{ }^{\circ} \mathrm{C}$ for $10 \mathrm{~s}, 60{ }^{\circ} \mathrm{C}$ for $30 \mathrm{~s}, 72{ }^{\circ} \mathrm{C}$ for $25 \mathrm{~s}$ and $78{ }^{\circ} \mathrm{C}$ for $2 \mathrm{~s}$. Fluorescence was quantified before and after the incubation at $78{ }^{\circ} \mathrm{C}$ to monitor the formation of primer dimers. The mRNA for $\beta$-tubulin was used as a control. A reaction mixture without reverse transcriptase was also used as a control to confirm the absence of genomic DNA contamination. Amplification of a single DNA fragment was confirmed by melting curve analysis and gel electrophoresis of the PCR products. Averages and standard errors of relative expression levels were calculated from PCR results for three independently synthesized cDNAs. Primer sequences used in expression analyses are listed in Additional file 3.

\section{Sequencing analysis of FT2 $a$ and SORE-1}

cDNAs from the three cultivars, TK780 and Hidaka 4 were used to sequence the FT2 $a$ coding regions. Each of the two FT2 $a$ genomic regions (the $5^{\prime}$ upstream region and the genic to $3^{\prime}$ downstream region) was divided into three parts, which were amplified from total DNA with KOD FX polymerase (Toyobo Life Science, Osaka, Japan) and sequenced. Genome walking with a BD Genome-Walker Universal kit (Takara Clontech, Otsu, Japan) was used to sequence the first intron of $\mathrm{TO}$, in which SORE-1 was inserted. According to the manufacturer's instructions, we constructed four kinds of genomic libraries by digesting total DNA from TO in separate reactions with four blunt-end endonucleases (DraI, EcoRV, PuvII, and StuI) and ligating the ends of the digested DNA to an adaptor sequence. Nested PCR was performed for each library using adaptor primers and gene-specific primers. The inserted SORE-1 was then amplified with the forward primer in intron 1 and the reverse primer in intron 2 , and the resultant amplicon was used for PCR amplification of each of five divided regions of SORE-1 to obtain the whole sequence 
(Additional file 4). The amplified fragments were sequenced directly or were first cloned into a pGEM-T Easy vector (Promega, Madison, WI, USA) and then sequenced. Sequence analysis was performed by using a BigDye Terminator v. 3.1 Cycle Sequencing kit and an ABI PRISM 3100 Avant Genetic Analyzer (Applied Biosystems Japan, Tokyo, Japan) according to the manufacturer's instructions. A BLAST search of the NCBI genome database and PLACE [47] analysis were carried out to detect sequences homologous to the fragment identified by genome-walking and possible cis-elements in the first intron of FT2a. Primer sequences used in genome sequencing are listed in Additional file 4.

\section{Genotyping for DNA polymorphisms in the FT2a genomic region and maturity loci}

DNA markers were developed to detect a 10-bp deletion in the $5^{\prime}$ UTR and the insertion of SNP \#17 and SORE-1 in the first intron. For the 10-bp deletion, the primers 5'-GGAATCGAGGCTATTGACTA-3' and 5'-CTTCC ACTAGGCATGGGATA-3' were used. For SORE-1, two forward primers, 5'-GCTCTCTCTCTTCCACTCTCT AGATGG-3' (in the long terminal repeat [LTR] of SORE-1) and 5'-ACCCTCTCAAGTGGACATGT-3' (in the first $F T 2 a$ intron), and the common reverse primer 5' -CTAGGTGCATCGGGATCAAC-3' (in the second FT2 $a$ exon) were used. To identify the SNP, a dCAPS marker was developed: PCR was performed with the primers 5' -TTCAAACAATCTCATAATTATGAGT-3' and 5 '-TAATAGTAGTATGGATGGTCAAA- 3 ', and the amplified products were digested with Hinfl. The PCR reaction and detection of amplified fragments were performed as described above. The genotyping for the $E 1, E 3$, and $E 4$ loci was performed using allele-specific DNA markers as described [18, 29]. Primers, PCR conditions and expected fragment sizes are presented in Additional file 5 .

\section{Methylation analysis}

Genomic DNA was extracted from trifoliate leaves of 20day-old plants of NILs for the FT2a-TO and FT2a-HY alleles, grown under SD. DNA samples were digested with McrBC (Takara Bio, Otsu, Japan). Digested and undigested samples were used for semi-quantitative PCR amplification of different regions of FT2a genomic and SORE-1 regions. Primer sequences used in methylation analyses are listed in Additional file 6.

\section{Availability of supporting data}

All supporting data can be found within the manuscript and its additional files. FT2 $a$ genomic sequences of Harosoy, Toyomusume and Hayahikari were deposited in the DNA Data Bank of Japan (DDBJ) under the accession numbers LC086649, LC086650 and LC086651, respectively.

\section{Additional files}

Additional file 1: DNA polymorphisms detected in the FT2a genomic region in four soybean cultivars or breeding lines and a wild soybean accession. (PDF $31 \mathrm{~kb}$ )

Additional file 2: Diurnal expression patterns of FT2a. (PDF $77 \mathrm{~kb}$ )

Additional file 3: Sequences of primers used in expression and RNA processing analyses of $F T 2 a$. (PDF $88 \mathrm{~kb}$ )

Additional file 4: Genomic positions and sequences of primers used in sequencing of the FT2a genomic region and Ty1/copia-like retrotransposon, SORE-1. (PDF $130 \mathrm{~kb}$ )

Additional file 5: Primers, PCR conditions and amplified fragment sizes for allele-specific DNA markers at maturity loci. (PDF $108 \mathrm{~kb}$ )

Additional file 6: Sequences of primers used in methylation analysis of $F T 2 a$. (PDF $129 \mathrm{~kb}$ )

\section{Abbreviations}

QTL: Quantitative trait locus; NIL: Near-isogenic line; RIL: Recombinant inbred line; PCR: Polymerase chain reaction; qRT-PCR: Quantitative real-time PCR; dCAPS: Derived cleaved amplified polymorphic sequence; FT: FLOWERING LOCUS T; DAS: Days after sowing; DAE: Days after emergence;

TE: Transposable element.

\section{Competing interests}

The authors declare that they have no competing interests.

\section{Authors' contributions}

CZ, FK, BL and JA designed and coordinated the study. JZ and JA carried out field experiments and phenotyping. MS and SW carried out mapping and statistical analysis, and developed near-isogenic lines. CZ, RT and MX conducted sequencing analyses. CZ and TY analyzed the gene expression and RNA processing. CZ and AK conducted methylation analyses and the data interpretation. CZ, SW, AK and JA drafted the manuscript with edits from FK, BL and TY. All authors read and approved the final manuscript.

\section{Acknowledgments}

This work was supported in part by Grants-in-Aid for Scientific Research from the Ministry of Education, Culture, Sports, Science, and Technology of Japan (23380001) to J Abe; by a grant from the Ministry of Agriculture, Forestry and Fisheries of Japan (Genomics-based Technology for Agricultural Improvement, SFC1003) to S Watanabe and T Yamada; by the Natural Science Foundation of China (31430065, 31371643 and 31571686); the Open Foundation of the Key Laboratory of Soybean Molecular Design Breeding, Chinese Academy of Sciences; the Strategic Action Plan for Science and Technology Innovation of the Chinese Academy of Sciences (XDA08030108) to B Liu and F Kong.

\section{Author details}

${ }^{1}$ Research Faculty of Agriculture, Hokkaido University, Sapporo, Hokkaido 060-8589, Japan. ²Faculty of Agriculture, Saga University, Saga 840-0027, Japan. ${ }^{3}$ The Key Laboratory of Soybean Molecular Design Breeding, Northeast Institute of Geography and Agroecology, Chinese Academy of Sciences, Harbin 150081, China.

Received: 16 October 2015 Accepted: 6 January 2016 Published online: 19 January 2016

References

1. Andrés F, Coupland $G$. The genetic basis of flowering responses to seasonal cues. Nat Rev Genet. 2012;13:627-39.

2. Itoh $\mathrm{H}$, Izawa $\mathrm{T}$. The coincidence of critical day length recognition for florigen gene expression and floral transition under long-day conditions in rice. Mol Plant. 2013;6:635-49.

3. Pin PA, Nilsson O. The multifaceted roles of FLOWERING LOCUS T in plant development. Plant Cell Environ. 2012;35:1742-55.

4. Watanabe S, Harada K, Abe J. Genetic and molecular bases of photoperiod responses of flowering in soybean. Breed Science. 2012;61:531-43.

5. Bernard RL. Two genes for time of flowering in soybeans. Crop Sci. 1971;11: 242-4. 
6. Buzzell RI. Inheritance of a soybean flowering response to fluorescentdaylength conditions. Can J Genet Cytol. 1971;13:703-7.

7. Buzzell RI, Voldeng HD. Inheritance of insensitivity to long day length. Soybean Genet Newsl. 1980;7:26-9.

8. McBlain BA, Bernard RL. A new gene affecting the time of flowering and maturity in soybean. J Hered. 1987;78:160-2.

9. Bonato ER, Vello NA. E6, a dominant gene conditioning early flowering and maturity in soybeans. Genet Mol Biol. 1999;22:229-32.

10. Cober ER, Voldeng HD. A new soybean maturity and photoperiod-sensitivity locus linked to E1 and T. Crop Sci. 2001:41:698-701.

11. Cober ER, Molnar SJ, Charette M, Voldeng HD. A new locus for early maturity in soybean. Crop Sci. 2010;50:524-7.

12. Kong F, Nan H, Cao D, Li Y, Wu F, Wang J, et al. A new dominant gene E9 conditions early flowering and maturity in soybean. Crop Sci. 2014;154:1220-31.

13. Ray JD, Hinson K, Mankono EB, Malo FM. Genetic control of a long-juvenile trait in soybean. Crop Sci. 1995;35:1001-6.

14. Liu B, Fujta T, Yan ZH, Sakamoto S, Xu D, Abe J. QTL mapping of domesticationrelated traits in soybean (Glycine max). Ann Bot. 2007;100:1027-38.

15. Xia Z, Watanabe S, Yamada T, Tsubokura Y, Nakashima H, Zhai H, et al. Positional cloning and characterization reveal the molecular basis for soybean maturity locus E1 that regulates photoperiodic flowering. Proc Natl Acad Sci USA. 2012;109:E2155-64.

16. Upadhyay AP, Summerfield RH, Ellis RH, Roberts EH, Qi A. Variation in the durations of the photoperiod-sensitive and photoperiod-insensitive phases of development to flowering among eight maturity isolines of soyabean [Glycine max (L.) Merrill]. Ann Bot. 1994;74:97-101.

17. Watanabe S, Tajuddin T, Yamanaka N, Hayashi M, Harada K. Analysis of QTLS for reproductive development and seed quality traits in soybean using recombinant inbred lines. Breed Sci. 2004:54:399-407.

18. Tsubokura Y, Watanabe S, Xia Z, Kanamori H, Yamagata H, Kaga A, et al. Natural variation in the genes responsible for maturity loci E1, E2, E3 and E4 in soybean. Ann Bot. 2014;113:429-41.

19. Watanabe S, Xia Z, Hideshima R, Tsubokura Y, Sato S, Harada K. A mapbased cloning strategy employing a residual heterozygous line reveals that the GIGANTEA gene is involved in soybean maturity and flowering. Genetics. 2011;188:395-407.

20. Watanabe S, Hideshima R, Xia Z, Tsubokura Y, Sato S, Nakamoto Y, et al. Map-based cloning of the gene associated with the soybean maturity locus E3. Genetics. 2009;182:1251-62.

21. Liu B, Kanazawa A, Matsumura H, Takahashi R, Harada K, Abe J. Genetic redundancy in soybean photoresponses associated with duplication of the phytochrome A gene. Genetics. 2008;180:995-1007.

22. Thakare D, Kumudini S, Dinkins RD. The alleles at the E1 locus impact the expression pattern of two soybean FT-like genes shown to induce flowering in Arabidopsis. Planta. 2011;234:933-43.

23. Wang Z, Zhou Z, Liu Y, Liu T, Li Q, Ji Y, et al. Functional evolution of phosphatidylethanolamine binding proteins in soybean and Arabidopsis. Plant Cell. 2015;27:323-36.

24. Kong F, Liu B, Xia Z, Sato S, Kim BM, Watanabe S, et al. Two coordinately regulated homologs of FLOWERING LOCUS T are involved in the control of photoperiodic flowering in soybean. Plant Physiol. 2010;154:1220-31.

25. Fan C, Hu R, Zhang X, Wang X, Zhang W, Zhang Q, et al. Conserved CO-FT regulons contribute to the photoperiod flowering control in soybean. BMC Plant Biol. 2014;14:9.

26. Sun H, Jia Z, Cao D, Jiang B, Wu C, Hou W, et al. GmFT2a, a soybean homolog of FLOWERING LOCUS T, is involved in flowering transition and maintenance. PLoS One. 2011:6:e29238.

27. Nan H, Cao D, Zhang D, Li Y, Lu S, Tang L, et al. GmFT2a and GmFT5a redundantly and differentially regulate flowering through interaction with and upregulation of the bZIP transcription factor GMFDL19 in soybean. PLoS One. 2014:9:e97669.

28. Xu M, Yamagishi N, Zhao C, Takeshima R, Kasai M, Watanabe S, et al. Soybean-specific maturity gene E1 family of floral repressors controls nightbreak responses through down-regulation of FLOWERING LOCUS T orthologs. Plant Physiol. 2015;168:1735-46

29. Xu M, Xu Z, Liu B, Kong F, Tsubokura Y, Watanabe S, et al. Genetic variation in four maturity genes affects photoperiod insensitivity and PHYA-regulated post-flowering responses of soybean. BMC Plant Biol. 2013;13:91.

30. Tsubokura Y, Matsumura H, Xu M, Liu B, Nakashima H, Anai T, et al. Genetic variation in soybean at the maturity locus $E 4$ is involved in adaptation to long days at high latitudes. Agronomy. 2013;3:117-34.
31. Wu F, Price BW, Haider W, Seufferheld G, Nelson R, Hanzawa Y. Functional and evolutionary characterization of the CONSTANS gene family in short-day photoperiodic flowering in soybean. PLoS One. 2014;9:e85754.

32. Zhang Q, Li H, Li R, Hu R, Fan C, Chen F, et al. Association of the circadian rhythmic expression of GmCRY1a with a latitudinal cline in photoperiodic flowering of soybean. Proc Natl Acad Sci USA. 2008;105:21028-33.

33. Matsumura H, Kitajima H, Akada S, Abe J, Minaka N, Takahashi R. Molecular cloning and linkage mapping of cryptochrome multigene family in soybean. Plant Genome. 2009;2:1-11.

34. Li F, Zhang $X$, Hu R, Wu F, Ma J, Meng Y, et al. Identification and molecular characterization of FKF1 and G/ homologous genes in soybean. PLoS One. 2013:8:e79036.

35. Hu Q, Jin Y, Shi H, Yang W. GmFLD, a soybean homolog of the autonomous pathway gene FLOWERING LOCUS D, promotes flowering in Arabidopsis thaliana. BMC Plant Biol. 2014:14:263.

36. Jia Z, Jiang B, Gao X, Yue Y, Fei Z, Sun H, et al. GmFULa, a FRUITFULL homolog, functions in the flowering and maturation of soybean. Plant Cell Rep. 2015;34:121-32.

37. Lu Q, Zhao L, Li D, Hao D, Zhan Y, Li W. A GmRAV ortholog is involved in photoperiod and sucrose control of flowering time in soybean. PLoS One. 2014:9:e89145.

38. Zhong X, Dai X, Xu J, Wu H, Liu B, Li H. Cloning and expression analysis of GmGAL1, SOC1 homolog gene in soybean. Mol Biol Rep. 2012;39:6967-74.

39. Na X, Jian B, Yao W, Wu C, Hou W, Jiang B, et al. Cloning and functional analysis of the flowering gene GmSOC1-like, a putative SUPPRESSOR OF OVEREXPRESSION CO1/AGAMOUS-LIKE 20 (SOC1/AGL20) ortholog in soybean. Plant Cell Rep. 2013;32:1219-29.

40. Zhao X, Cao D, Huang Z, Wang J, Lu S, Xu Y, et al. Dual functions of GMTOE4a in the regulation of photoperiod-mediated flowering and plant morphology in soybean. Plant Mol Biol. 2015;88:343-55.

41. Xue ZG, Zhang XM, Lei CF, Chen XJ, Fu YF. Molecular cloning and functional analysis of one ZEITLUPE homolog GmZTL3 in soybean. Mol Biol Rep. 2012;39:1411-8.

42. Zhang J, Song Q, Cregan PB, Nelson RL, Wang X, Wu J, et al. Genome-wide association study for flowering time, maturity dates and plant height in early maturing soybean (Glycine max) germplasm. BMC Genomics. 2015;16:217.

43. Jiang B, Yue Y, Gao Y, Ma L, Sun S, Wu C, et al. GmFT2a polymorphism and maturity diversity in soybeans. PLoS One. 2013:8:e77474.

44. Tanaka Y, Tomita K, Yumoto S, Kurosaki H, Yamazaki H, Suzuki C, et al. A new soybean variety Yukihomare. Bull Hokkaido Prefect Agric Exp Stn. 2003; 84:13-24.

45. Schmutz J, Cannon SB, Schlueter J, Ma J, Mitros T, Nelson W, et al. Genome sequence of the palaeopolyploid soybean. Nature. 2010;463:178-83.

46. Kanazawa A, Liu B, Kong F, Arase S, Abe J. Adaptive evolution involving gene duplication and insertion of a novel Ty1/copia-like retrotransposon in soybean. J Mol Evol. 2009;69:164-75.

47. Higo $\mathrm{K}$, Ugawa $\mathrm{Y}$, Iwamoto M, Korenaga T. Plant cis-acting regulatory DNA elements (PLACE) database. Nucl Acids Res. 1999;27:297-300.

48. Varagona MJ, Purugganan M, Wessler SR. Alternative splicing induced by insertion of retrotransposons into the maize waxy gene. Plant Cell. 1992;4: $811-20$.

49. Iwata H, Gaston A, Remay A, Thouroude T, Jeauffre J, Kawamura K, et al. The TFLI homologue KSN is a regulator of continuous flowering in rose and strawberry. Plant J. 2012;69:116-25.

50. Liu J, He Y, Amasino R, Chen X. siRNAs targeting an intronic transposon in the regulation of natural flowering behavior in Arabidopsis. Genes Dev. 2004;18:2873-8.

51. Shibuya K, Fukushima S, Takatsuji H. RNA-directed DNA methylation induces transcriptional activation in plants. Proc Natl Acad Sci USA. 2009:106:1660-5.

52. Deng S, Chua NH. Inverted-repeat RNAs targeting $F T$ intronic regions promote FT expression in Arabidopsis. Plant Cell Physiol. 2015;56:1667-78.

53. Michaels SD, Amasino RM. FLOWERING LOCUS C encodes a novel MADS domain protein that acts as a repressor of flowering. Plant Cell. 1999;11: 949-56.

54. Michaels SD, He Y, Scortecci KC, Amasino RM. Attenuation of FLOWERING LOCUS C activity as a mechanism for the evolution of summer-annual flowering behavior in Arabidopsis. Proc Natl Acad Sci USA. 2003;100:10102-7.

55. Fehr WR, Caviness CE, Burmood DT, Pennington JS. Stage of development descriptions for soybeans, Glycine max (L.) Merrill. Crop Sci. 1971;11:929-31.

56. Doyle JJ, Doyle JL. Isolation of plant DNA from fresh tissue. Focus. 1990;12: $13-5$. 
57. Cregan PB, Jarvik T, Bush AL, Shoemaker RC, Lark KG, Kahler AL, et al. An integrated genetic linkage map of the soybean genome. Crop Sci. 1999;39: 1464-90.

58. Qj S, Jia GF, Zhu YL, Grant D, Nelson RT, Hwang EY, et al. Abundance of SSR motifs and development of candidate polymorphic SSR markers (BARCSOYSSR_1.0) in soybean. Crop Sci. 2010;50:1950-60.

59. Napoli C, Lemieux C, Jorgensen R. Introduction of a chimeric chalcone synthase gene into Petunia results in reversible co-suppression of homologous genes in trans. Plant Cell. 1990;2:279-89.

60. Dwiyanti MS, Yamada T, Sato M, Abe J, Kitamura K. Genetic variation of $Y^{-}$ tocopherol methyltransferase gene contributes to elevated a-tocopherol content in soybean seeds. BMC Plant Biol. 2011;11:152.

Submit your next manuscript to BioMed Central and we will help you at every step:

- We accept pre-submission inquiries

- Our selector tool helps you to find the most relevant journal

- We provide round the clock customer support

- Convenient online submission

- Thorough peer review

- Inclusion in PubMed and all major indexing services

- Maximum visibility for your research

Submit your manuscript at www.biomedcentral.com/submit
Biomed Central 